\title{
Analyse et sécurité santaire
}

Oléagineux, Corps Gras, Lipides. Volume 10, Numéro 4, JUILLET-AOÛT 2003, Journées de Printemps de l'AFECG, Paris, 1-2 avril 2003

Auteur(s) : André POUZET , Président de l'AFECG.

\section{ARTICLE}

\section{Auteur(s) : André POUZET}

\section{Président de l'AFECG}

L'idée d'organiser deux journées de conférence sur les Innovations et les Problématiques Actuelles dans l'analyse des oléagineux et des corps gras a été acceptée par le Conseil d'Administration de I'Association Française pour l'Etude des Corps Gras (AFECG) sur proposition de Jean-Luc Perrin ${ }^{\underline{1}}$.

Il s'agit là d'une excellente idée, puisque l'analyse est un élément fondamental de l'activité de notre secteur, et qu'elle n'avait pas été abordée en tant que telle depuis plus de 10 ans.

L'analyse est au cour de notre ativité pour deux bonnes raisons au moins :

- d'abord, elle caractérise le résultat de la mise en cuuvre de process industriels; cet énoncé relativement simpliste doit être pris avec beaucoup de précaution, puisqu'il s'agit en fait de caractériser un produit par plusieurs analyses n'ayant pas toutes la même importance pour qualifier le résultat d'un process industriel. Des échanges entre les scientifiques qui rendent possible la mise en ceuvre de techniques analytiques de plus en plus performantes, les industriels pour qui les enjeux qualitatifs sont de plus en plus forts et les équipementiers qui doivent anticiper l'évolution des besoins, sont donc nécessaires, et - parmi d'autres - les Journées organisées par l'AFECG fournissent un cadre pour faciliter ces rencontres et échanges.

- De plus, elle apporte des garanties, avec leur domaine d'incertitudes, aux maillons successifs de la filière et finalement au consommateur. Là encore, il convient de préciser : pour les spécialistes du domaine de l'analyse, le point important est certainement le domaine d'incertitude, alors que le consommateur aura plutôt tendance à considérer la valeur annoncée sur une étiquette comme unique et définitive. Que l'on pense par exemple à la difficulté que l'on rencontre parfois à expliciter en dehors du cercle des initiés - les notions fondamentales que sont la limite de détection ou la limite de quantification. Des réunions comme celle-ci permettent à chacun de réflechir aux interprétations qui sont proposées et à la meilleure façon de communiquer les résultats vis-à-vis des tiers.

Pourquoi aborder ce thème en 2003 ? Une réponse simple est fournie par une évocation rapide des évolutions importantes que chacun a pu constater au cours des dernières années :

- évolution d'abord dans le domaine de l'instrumentation, de la miniaturisation et plus généralement de la technologie analytique (par exemple les développements des utilisations de la RMN, de la spectrophotométrie, sans oublier les couplages en chromatographie); 
- renforcement ensuite de la normalisation et de la réglementation, qui peuvent être considérées comme des contraintes pour les entreprises, mais qui peuvent également constituer des atouts quand elles sont intelligement conçues. Depuis de nombreuses années, I'ONIDOL (Interprofession des Oléagineux), la FNCG (Fédération Nationale des Industries des Corps Gras) et les Centres Techniques de la filière, CETIOM et ITERG) apportent leur soutien aux opérations de normalisation gérées par I'AFNOR, le CEN et I'ISO, tant il est vrai qu'il n'y a pas d'échanges commerciaux fiables sans analyse fiable et qu'il n'y a pas d'analyse fiable sans une normalisation définie consensuellement entre les acteurs.

- médiatisation enfin, toujours plus aigue autour de certains sujets, pas forcément nouveaux mais ramenés à la surface par les progrès analytiques. On pense à certains sujets récurrents tels que les dioxines ou les acrylamides.

Le programme des Journées de Printemps de l'AFECG, dont ce numéro d'OCL présente les compterendus, a repris ces différents aspects :

- expression des attentes des industriels, avec la présentation de Pierre-Yves Vigneron,

- présentation des innovations récentes dans le domaine de la technologie analytique avec les compte-rendus de Marc Meurens et de Patrick Mielle respectivement sur la spectrophotométrie et les nez électroniques,

- évolution de la normalisation et des circuits d'essais, des réglementations communautaires et nationales, avec les compte-rendus de Marie Lafargue, Odile Morin et Alain Quinsac,

- résultats récents rapportés par D. Fraisse, Christian Gertz, Marie-Paule Herry, Florence Lacoste et Jean-François Narbonne sur les contaminants tels que dioxines, acrylamide, HAP, pesticides, ou mycotoxines,

- détection de l'adultération des huiles végétales avec un état de l'art présenté par Denis Ollivier et les problèmes analytiques liés à l'évolution de la réglementation en chocolaterie discutée par Henri Chaveron.

La qualité des intervenants sur ces sujets scientifiques d'actualité contribue à la richesse de ce dossier que les lecteurs d'OCL apprécieront, nous l'espérons.

${ }^{1}$ Jean-Luc Perrin (UNILEVER) a également animé le Comité Scientifique des Journées de Printemps 2003, avec la participation de Florence Lacoste (ITERG), de Michel Blanc (EXPERAGRO), d'Alain Quinsac (CETIOM) et de Pierre-Yves Vigneron (LESIEUR). Qu'ils trouvent ici l'expression des remerciements de l'AFECG. 